\title{
Electron emission and positron production in deep inelastic heavy-ion reactions
}

\author{
U. Müller and G. Soff \\ Gesellschaft für Schwerionenforschung mbH, D-6100 Darmstadt 11, Federal Republic of Germany \\ J. Reinhardt, T. de Reus, B. Müller, and W. Greiner \\ Institut für Theoretische Physik der Johann Wolfgang Goethe-Universität, \\ D-6000 Frankfurt am Main, Federal Republic of Germany
}

(Received 7 February 1984)

\begin{abstract}
Atomic excitations are used to obtain information on the course of a nuclear reaction. Employing a semiclassical picture we calculate the emission of $\delta$ electrons and positrons in deep inelastic nuclear reactions for the example of $U+U$ collisions incorporating nuclear trajectories resulting from two different nuclear friction models. The emission spectra exhibit characteristic deviations from those expected for elastic Coulomb scattering. The theoretical probabilities are compared with recent experimental data by Backe et al. A simple model is used to estimate the influence of a threebody breakup of the compound system upon atomic excitations.
\end{abstract}

\section{THE DESCRIPTION OF ELECTRONIC EXCITATIONS}

In collisions of very heavy ions superheavy quasiatomic systems are created, where the electrons experience for a period of time $T \sim 10^{-20} \mathrm{~s}$ the combined nuclear charge $Z=Z_{P}+Z_{T}$ of the projectile and the target nucleus. The resulting enormous binding energies and the highmomentum components of the wave functions necessitate a relativistic treatment of the dynamical behavior of the electrons. Under these conditions excitation processes of electrons and positrons are appropriately described in a semiclassical picture based on the time-dependent twocenter Dirac equation $(\hbar=c=1)$

$$
i \frac{\partial}{\partial t} \Phi_{i}(\overrightarrow{\mathbf{R}}(t))=H_{\mathrm{TCD}}(\overrightarrow{\mathbf{R}}(t)) \Phi_{i}(\overrightarrow{\mathbf{R}}(t))
$$

where $H_{\mathrm{TCD}}$ is the relativistic two-center Hamiltonian depending on the time-dependent classical internuclear separation $\overrightarrow{\mathbf{R}}(t)$. Equation (1) determines the motion of a single electron, initially in state $i$, in the external timevarying electromagnetic field. Since for nonrelativistic bombarding energies the inner-shell electrons move quasiadiabatically, the total electron wave function is expanded into the set of adiabatic eigenstates $\phi_{j}$ of the Hamiltonian:

$$
\Phi_{i}(\overrightarrow{\mathbf{R}}(t))=\sum_{j} a_{i j}(t) \phi_{j}(\overrightarrow{\mathbf{R}}(t)) \exp \left[-i \chi_{j}(t)\right] .
$$

The expansion includes the bound states as well as an integration over continuum states of positive and negative frequencies. The phase factors $\chi_{j}$ are chosen conveniently to eliminate the diagonal matrix elements of the Hamiltonian $H_{\mathrm{TCD}}$. Inserting the expansion (2) into (1) and projecting with stationary eigenfunctions we obtain a set of coupled differential equations for the amplitudes $a_{i j}(t)$,

$$
\begin{gathered}
\dot{a}_{i j}(t)=-\sum_{k \neq j} a_{i k}(t)\left\langle\phi_{j}\left|\partial / \partial t+i H_{\mathrm{TCD}}\right| \phi_{k}\right\rangle \\
\times \exp \left[i\left(\chi_{j}-\chi_{k}\right)\right],
\end{gathered}
$$

with the initial condition $a_{i j}(-\infty)=\delta_{i j}$.

Since multistep excitation processes are crucial for a quantitative understanding of inner-shell vacancy formation in scattering processes involving very heavy ions, one has to integrate the coupled equations for the electron occupation amplitudes rigorously. ${ }^{1-3}$

The fate of a single electron during the collision is determined by the occupation amplitudes. Considering interactions between various electrons through a mean field only, excitations of the many-electron system can be described by the one-electron transition probabilities as well. After the collision the number of particles in a state $p$ above the Fermi level $F$, up to which the quasimolecular levels were filled initially, is (taking into account spin degeneracy)

$$
N_{p}=2 \sum_{k<F}\left|a_{k p}(+\infty)\right|^{2}, p>F .
$$

For the number of holes in a state $q$ below the Fermi level $F$ one has to calculate

$$
N_{q}=2 \sum_{k>F}\left|a_{k q}(+\infty)\right|^{2}, q<F .
$$

In particular, this result applies to pair creation, where a hole in the negative energy continuum after the collision corresponds to a positron. The methods and approximations used to solve the coupled channel equations (3) are described elsewhere. ${ }^{1-4}$ Here we want to stress three general features of our calculations:

(i) The multipole expansion of the two-center potential is restricted to its monopole $(l=0)$ term. ${ }^{1}$ This is identical to smearing out the nuclear charge in a spherical shell with radius $R / 2$ and a width of twice the nuclear radius. Due to the monopole restriction the time-derivative opera- 
tor $\partial / \partial t$ in (3) reduces to purely radial coupling $\dot{R}(\partial / \partial R)$.

(ii) Electron screening effects are taken into account within an adiabatic approximation to the time-dependent Hartree-Fock (TDHF) model. ${ }^{4}$ The basis states to be inserted in (2) are solutions of the static Hartree-Fock-Slater Hamiltonian at the given value of $R$. The time-dependent problem then is solved neglecting the influence of excitation processes on electron shielding, i.e., assuming the quasimolecule to be always in its ground state during the course of the collision. For the present calculations we have assumed that 50 electrons in the highest molecular states are missing. As for the two-center Coulomb potential, cf. (i), only the monopole part of the screening potential has been taken into account. All results have to be referred to these assumptions for the self-consistent potential, which is discussed in Ref. 4.

(iii) The potential coupling in (3), which acts only if the basis does not consist of exact eigenstates of the instantaneous Dirac Hamiltonian, is of particular importance for the description of positron creation in supercritical heavy-ion collisions $(Z \geq 173)$. For those systems the binding energy of the strongest bound state exceeds $2 m_{\mathrm{e}} c^{2}$. When the state becomes imbedded as a resonance in the lower continuum, a projection method can be applied $^{3}$ yielding a normalizable quasibound state $\phi_{R}$ at resonance energy $E_{R}$ as well as a new negative energy continuum $\widetilde{\phi}_{\mathrm{e}^{+}}$orthogonal to $\phi_{R}$. Since $\phi_{R}$ is not an eigenstate of the Hamiltonian, a hole prepared in $\phi_{R}$ will decay exponentially with a decay width

$$
\Gamma=2 \pi\left|\left\langle\widetilde{\phi}_{E_{\text {res }}}\left|H_{\mathrm{TCD}}\right| \phi_{R}\right\rangle\right|^{2} .
$$

The formalism thus naturally leads to the emergence of "induced" and "spontaneous" positron creation, the latter resulting from the presence of an unstable state $\phi_{R}$ in the expansion basis.

In the monopole approximation states of different angular momentum do not couple. For the present calculations, we have solved the system of differential equations including up to 8 bound states and $\sim 17$ states in the upper continuum, separately for the two angular momentum channels $\kappa=+1$ and $\kappa=-1\left(s_{1 / 2}\right.$ and $p_{1 / 2}$, respectively), which are dominant for the production of high energy $\delta$ electrons and of positrons. Since the coupling between positron states is small, it is sufficient to include only one positron state at a time in the calculation of the positron spectrum, i.e., the lower continuum can be coupled in perturbation theory.

\section{EMISSION OF $\delta$ ELECTRONS AND POSITRONS IN DEEP-INELASTIC REACTIONS}

In the following we will discuss the emission of $\delta$ electrons and the creation of positrons due to the timevarying electric field in heavy-ion collisions with nuclear contact and, in particular, in deep-inelastic nuclear reactions.

In Refs. 5-7 we used within our semiclassical description of heavy-ion collisions a very simplified approach for the nuclear motion. The schematic model facilitated a systematic study of the time delay effect. Moreover, we had taken advantage of its unrestricted applicability for the limit of very long reaction times, for which up to now no nuclear model calculations exist.

For the description of a given experiment, however, it is more convincing to adopt trajectories calculated from a nuclear model which is consistent with the elastic and inelastic heavy-ion scattering data. Deep-inelastic reactions have been discussed in terms of many models with different degrees of refinement.

In the following we will employ two different macroscopic friction models for the nuclear motion in $\mathbf{U}+\mathbf{U}$ collisions. The first model, proposed by Birkelund et al. ${ }^{8}$ (hereafter denoted by I) is based on the proximity nuclear potential of Blocki et al. ${ }^{9}$ and the one-body nuclear friction in the proximity formalism of Randrup. ${ }^{10}$ We have neglected the deviations of the Coulomb potential from the point-charge value due to the nuclear extension as given, e.g., by Bondorf et al. ${ }^{11}$ The model of Birkelund et $a l .{ }^{8}$ incorporates nuclear intrinsic rotation and has a set of dynamical variables $\left\{R, P, \vartheta_{T}, l_{T}, \vartheta_{P}, l_{P}\right\}$, i.e., the internuclear distance and the orientation angles of the individual nuclei and their corresponding conjugate momenta, for which the classical equations of motion are solved. As an alternative we employ the nuclear trajectories of Schmidt et al. ${ }^{12}$ who have proposed a macroscopic friction model (model II), which in a simple way accounts for neck formation in the separating system. Thereby one is able to explain the experimentally observed energy loss, where up to $\sim 30 \%$ energy dissipation for $b \sim 0$ can be achieved.

Strong deviations from a Coulomb trajectory and, at the same time, increased reaction times are found in both models. This is demonstrated in Fig. 1 for the case of head-on collisions $(b=0)$ of $U+U$ at a bombarding energy of $E_{\text {lab }}=7.5 \mathrm{MeV} /$ nucleon. Here model II predicts delay times up to $\Delta T \sim 1.1 \times 10^{-21} \mathrm{~s}$ defined with respect to the point of nuclear separation. We note that it is impossible to give an unambiguous definition of the time delay in the presence of energy dissipation: From the atomic

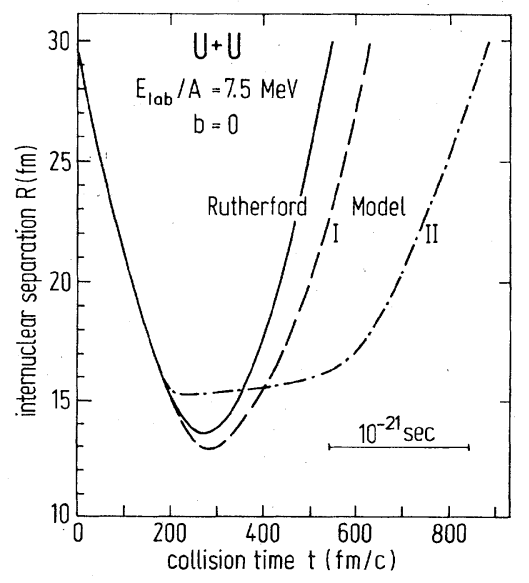

FIG. 1. The nuclear trajectories of central $U+U$ collisions at laboratory energy $7.5 \mathrm{MeV} /$ nucleon resulting from the friction models of Ref. 8 (model I, dashed line) and Ref. 12 (model II, dash-dotted line) compared with pure Coulomb scattering (full line). 
point of view the collision time of the two nuclei seems to be enhanced due to the reduced radial velocity in the outgoing channel. Thus one might introduce an interaction sphere, depending on the considered atomic excitation process, and define the delay time $\Delta T$ with reference to this distance.

The influence of such a modification of the nuclear motion on the atomic excitation processes is of particular interest. However, it is obvious from the findings within the schematic trajectory model that the spectra of emitted electrons and positrons will not be altered radically due to the rather short reaction times except for the influence of nuclear trajectories of model II on $\delta$-electron spectra in very central collisions as discussed in the following. Positron spectra show a gradual enhancement at their maxima as well as a drift toward lower positron kinetic energies. ${ }^{6}$ The latter effect can be described in terms of destructive interferences between atomic excitations on the incoming and the outgoing path of the trajectory, while the first one is due to the spontaneous positron production mechanism in the $s_{1 / 2}$ channel. But it is also clear that delay times of the scale introduced by the friction models for the nuclear trajectory in deep-inelastic collisions are not long enough to cause the unambiguous emergence of a positron line ${ }^{6}$ as a united atom effect.

For different kinematic conditions (e.g., larger bombarding energies) or other colliding systems (like $\mathbf{U}+\mathrm{Pb}$, $\mathrm{U}+\mathrm{Cm}$ ) the described effects may be more pronounced: ${ }^{13}$ In $\mathrm{U}+\mathrm{Pb}$ the kinematic modifications due to the macroscopic friction model II lead to a narrowing of the spectrum in both $s$ - and $p_{1 / 2}$-state contributions since both are subcritical channels. In the $\mathrm{U}+\mathrm{Cm}$ system the contribution from the supercritical $s$ states grows faster than in $\mathbf{U}+\mathbf{U}$ due to the larger spontaneous decay width, thus providing a clearer signature for a prolonged reaction time.

The spectra of emitted $\delta$ electrons decrease considerably in their high-energy part compared with spectra calculated for Rutherford scattering, and their falloff is steeper. All these effects are established best for the innermost electrons which are extremely sensitive to the nuclear charge configuration and thus can be strongly influenced by the changed nuclear kinetics. Since the effect on the nuclear trajectories within these models is small for peripheral collisions, atomic excitation processes are disturbed most in central heavy-ion collisions.

The dependence on the impact parameter $b$ is demonstrated in Fig. 2 for the three models under discussion. $\delta$-electron spectra calculated for pure Rutherford scattering [part (a)] show the familiar increase with decreasing impact parameter. Applying model I [part (b)], emission probabilities (e.g., at $E_{\mathrm{e}^{-}} \sim 1500 \mathrm{keV}$ ) get reduced by half in central collisions and are rather weakly dependent on impact parameter for a broad region of $b \lesssim 7 \mathrm{fm}$. Using trajectories calculated within the framework of model II [part (c)], a drastic monotonic decrease in the $\delta$-electron probabilities is found when going to more central collisions, in complete contrast to the predictions of Rutherford scattering and also to nuclear reactions of the model I type. The steeper falloff of the spectra is due to destructive interferences between the excitation amplitudes on the incoming and outgoing path of the trajectory as anticipated for delayed collisions.

Recently experimental data have been published by Backe et al. ${ }^{14}$ concerning the energy spectra of $\delta$ electrons and positrons emitted in $\mathbf{U}+\mathbf{U}$ and $\mathrm{U}+\mathrm{Cm}$ collisions above the Coulomb barrier. To get a signature for close contact, the atomic excitations have been measured in coincidence with fission fragments, which were detected in a laboratory angular window of $\theta_{\mathrm{lab}}=40^{\circ} \pm 5^{\circ}$. (For the bombarding energy of $E_{\text {lab }}=5.9 \mathrm{MeV} /$ nucleon an angular window of $\theta_{\mathrm{lab}}=45^{\circ} \pm 10^{\circ}$ was used.)

For a quantitative comparison with the experiment one has to integrate the theoretical impact-parameterdependent spectra over all values of $b$ which lead to a nuclear reaction, weighted by the corresponding probability $w_{f}(b)$ to induce nuclear fission, and by the probability $w_{D}(b)$ to detect the fission residues within the experimentally given angular window

$$
d P_{\mathrm{e}^{ \pm}} / d E_{\mathrm{e}^{ \pm}}=\int b d b d P_{\mathrm{e}^{ \pm}}(b) / d E_{\mathrm{e}} \pm w_{f}(b) w_{D}(b) / \int b d b w_{f}(b) w_{D}(b)
$$

Values for the fission probabilities $w_{f}(b)$ can be derived ${ }^{13}$ from measurements of Freiesleben et al., ${ }^{15}$ who for 7.42 $\mathrm{MeV} /$ nucleon $\mathrm{U}+\mathrm{U}$ collisions investigated the elastic (plus quasielastic) angular scattering distribution compared to the averaged Mott cross section. The analysis yields a quarter point angle of $\theta_{1 / 4}=87.5^{\circ} \pm 2^{\circ}$, which corresponds to a classical distance of closest approach of $16.85 \mathrm{fm}$, fitting nicely ${ }^{15}$ into the systematics of strong absorption radii given by Birkelund and Huizenga. Although a procedure to determine $w_{f}(b)$ from these data is not unique, ${ }^{13}$ due to contributions from forward and backward scattering, the final results depend only slightly on the estimates used, i.e., $\delta$-electron and positron spectra change only slightly within the experimentally ${ }^{15}$ given boundaries.

The folding with the detection probability $w_{D}(\mathrm{~b})$ in (6) depends on the details of the experimental setup, in partic- ular on the acceptance criteria of the particle detectors. In the experiment of Backe et al. ${ }^{14}$ the detection probability was studied ${ }^{16}$ taking into account three and four body breakup. A Monte Carlo procedure was used to simulate different kinds of breakup (mass asymmetry, $Q$ value, direction) for each impact parameter. Although it carries a considerable uncertainty we found that the deduced ${ }^{16}$ experimental detection probability has no large influence on the calculation of $\delta$-electron and positron spectra. In consequence we will use $w_{D}=1$ everywhere in the following discussion, thus leaving the results independent of experimental details.

Figure 3 shows the experimental data ${ }^{14}$ for $\delta$-electron emission in 5.9 and $7.5 \mathrm{MeV} /$ nucleon $U+U$ collisions in comparison with theoretical results including electron shielding. The dots in Fig. 3(a) represent measurements in coincidence with elastic and quasielastic particle scatter- 

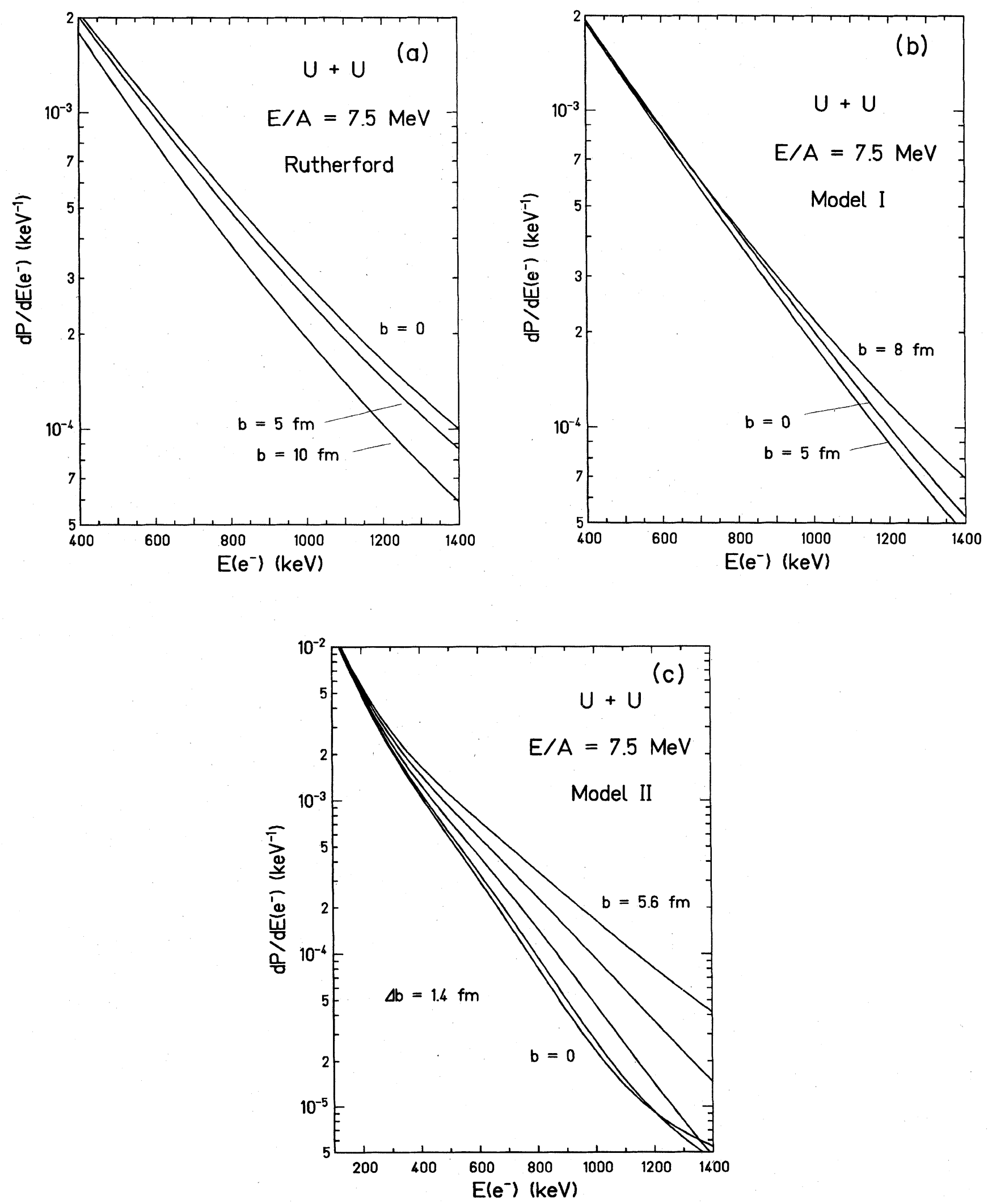

FIG. 2. Spectra of $\delta$ electrons emitted in $7.5 \mathrm{MeV} /$ nucleon $U+U$ collisions are shown for various fixed values of the impact parameter $b$. Trajectories resulting from pure Coulomb scattering (a), model I (b), and model II (c) were employed. Note the expanded scales in part (c). 

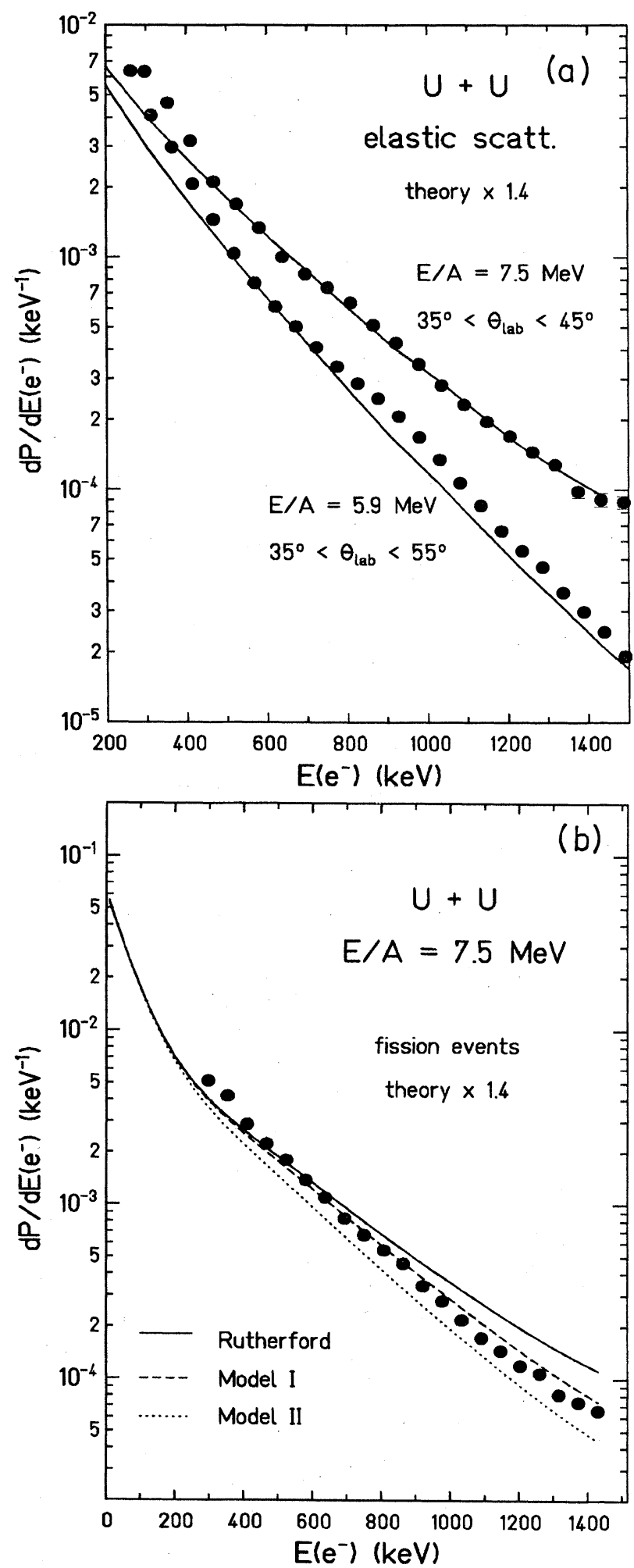

FIG. 3. Spectra of $\delta$ electrons emitted in 5.9 and 7.5 $\mathrm{MeV} /$ nucleon $\mathrm{U}+\mathrm{U}$ collisions measured by Backe et al. (Ref. 14) for elastic and quasielastic scattering (a) and in coincidence with nuclear fission residues (b). The experimental data are compared with calculations assuming Coulomb scattering (full lines) and friction model trajectories [dashed (Ref. 8) and dotted (Ref. 12) lines]. All calculated spectra are shifted up by a factor of 1.4 . ing. The full lines showing calculations for Rutherford scattering fit the data quite nicely in slope. However, the theoretical results had to be adjusted by a factor 1.4. The same adjustment was required to explain the $\delta$-electron data for both bombarding energies. The broad experimental distribution above the theoretical values for electron energies of $E_{\mathrm{e}^{-}} \sim 1000 \mathrm{keV}$ (at $E_{\mathrm{lab}}=5.9 \mathrm{MeV} /$ nucleon) stems from a possible $E 0$ conversion, ${ }^{14}$ which, however, seems to be negligible ${ }^{14}$ at $7.5 \mathrm{MeV} /$ nucleon.

$\delta$-electron spectra at $7.5 \mathrm{MeV} /$ nucleon bombarding energy taken in coincidence with fission products (i.e., following a nuclear reaction) fall off much more steeply [cf. Fig. 3(b)]. For comparison theoretical $\delta$-electron probabilities are shown where even for small impact parameters pure Rutherford scattering is assumed, thus simulating "transparent" nuclear matter (solid line), followed by a nuclear fission process. The observation of an increased slope of $d P / d E_{\mathrm{e}^{-}}$in collisions leading to fission is in agreement with theoretical expectations: From Figs. 2(b) and (c) it is obvious that for events with increasing inelasticity the spectra fall off more steeply due to destructive interference. This effect survives the integration over impact parameter [cf. Eq. (6)]. The dashed line displays a spectrum calculated with the modified trajectories of reaction model I (Birkelund et al. ${ }^{8}$ ), whereas the dotted line represents calculations for model trajectories of Schmidt et $a l .{ }^{12}$ (model II). Again all theoretical probabilities have been scaled by a common factor 1.4 .

In all calculations the trajectories of a binary system have been used, assuming that the fission process (which is delayed ${ }^{17}$ by $\sim 10^{-20}$ s) does not severely modify $\delta$ electron emission. While a full three-center calculation seems quite discouraging, it is possible to check the consequences of this assumption, again using the monopole approximation (see Sec. III).

The absolute values seem to favor the reaction model I (Ref. 8) (note, however, that these values are not so well understood, as demonstrated by the need for a scaling factor even in the case of elastic scattering). The slope of $\delta$ electron emission probabilities is reproduced much better by calculations based on the trajectories of model II. ${ }^{12}$ Analogous results have been obtained for a bombarding energy of $E_{\text {lab }}=8.4 \mathrm{MeV} /$ nucleon. Again the $\delta$-electron spectrum calculated for Rutherford scattering has a slope which is too flat to fit the experimental data. ${ }^{14}$ Predictions using model I miss both slope and absolute value. Model II leads to $\delta$-electron spectra having the correct slope, but again a scaling factor different from that which fits the elastic scattering data for $E_{\mathrm{lab}}=5.9$ and 7.5 $\mathrm{MeV} /$ nucleon has to be used.

Positron spectra measured and calculated under the same kinematical conditions as for the "deep-inelastic" $\delta$ electron spectra in Fig. 3(b), i.e., $U+U$ reactions at 7.5 $\mathrm{MeV} /$ nucleon bombarding energy, are presented in Fig. 4. The theoretical values have been reduced by a common factor $\sim 0.85$, which was adjusted to fit the experimental results at the maximum of the positron spectrum.

In Fig. 5 the experimental positron data ${ }^{14}$ for heavy-ion collisions of $U+U$ at $5.9,7.5$, and $8.4 \mathrm{MeV} /$ nucleon (the latter scaled up by a factor 3 for the sake of better separation of the curves) are collected and compared with 


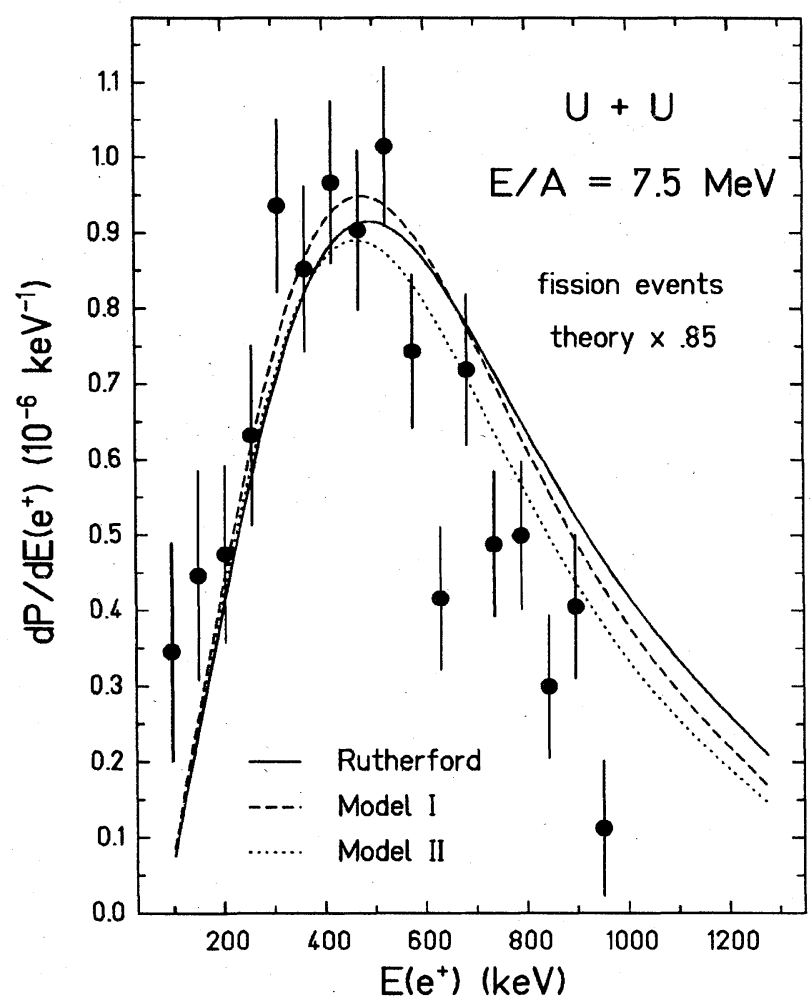

FIG. 4. The experimental positron spectrum (Ref. 14) for 7.5 $\mathrm{MeV} /$ nucleon $\mathrm{U}+\mathrm{U}$ collisions leading to fission in comparison with theoretical predictions based on Coulomb scattering and two friction models (Refs. 8 and 12). The theoretical spectra are shifted down by a factor of 0.85 .

theoretical results. For the lowest bombarding energy elastic scattering events have been measured; thus we have to compare with theoretical results for positrons emitted along a Rutherford scattering path. For all other collisions also nuclear trajectories derived from models I and II have been assumed.

The comparison between experimental data ${ }^{14}$ and theoretical positron spectra assuming Rutherford trajectories shows an enhancement of the experimental probabilities at kinetic energies near the maximum and a narrowing of the whole spectrum, in qualitative agreement with the theoretical results due to the friction model trajectories. Taking the data at face value, one may conclude that for a better agreement even longer delay times $\Delta T \sim 2 \times 10^{-21} \mathrm{~s}$ are required. This would stand in contrast to our conclusions from the $\delta$-electron spectra. We want to add a word of caution. In the course of the collision the nuclei can be excited by Coulomb and nuclear forces. In the final state virtually emitted photons with energy above $1022 \mathrm{keV}$ can undergo internal pair conversion. One has to subtract this background by simultaneously measuring the $\gamma$ spectrum and folding it with the conversion coefficients. Here one has to know-or assume-the $\gamma$-ray multipolarity. Up to now, all conclusions on positron production in heavy-ion collisions have relied on this procedure for background subtraction. Moreover, monopole conversion cannot be handled by this

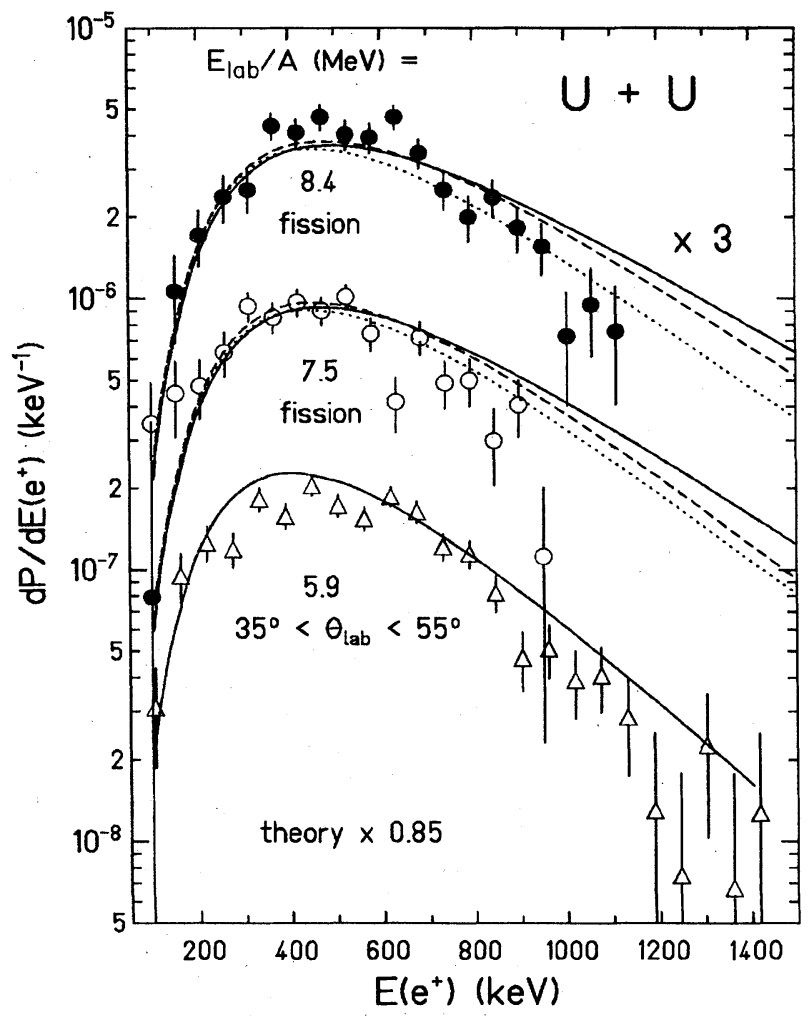

FIG. 5. Experimental spectra (Ref. 14) of positrons emitted in $\mathrm{U}+\mathrm{U}$ collisions at $5.9 \mathrm{MeV} /$ nucleon (elastic scattering) and at 7.5 and $8.4 \mathrm{MeV} /$ nucleon (in coincidence with nuclear fission). The data are compared with theoretical results based on Rutherford scattering (full lines) and two friction models [dashed (Ref. 8) and dotted (Ref. 12) lines]. All theoretical spectra have been reduced by a factor of 0.85 .

method. The high energy tail of the measured positron spectrum may be particularly affected by this problem. ${ }^{18}$

Thus it is difficult to draw definite conclusions from the positron data: Although the trend toward enhanced reaction time (steeper slope) at high impact energy is clearly observed, a quantitative explanation has not been achieved. Judging from the experimental data published up to now, the $\delta$-electron spectra seem to provide more reliable information on the reaction process due to the larger cross sections and thus smaller experimental error bars. To obtain a fully consistent picture of the reaction mechanism, however, the measurement of both types of emission spectra, as well as the investigation of $K$-hole production, ${ }^{19}$ is required.

\section{THE INFLUENCE \\ OF A NUCLEAR THREE-BODY BREAKUP ON ATOMIC EXCITATION PROCESSES}

In the preceding section we investigated the influence of modified nuclear trajectories in deep-inelastic heavy-ion reactions on atomic excitation processes. We assumed the fission process to happen at an internuclear distance large enough ${ }^{17}$ that it does not severely disturb processes like $\delta$-electron emission or positron creation. Here we want to 

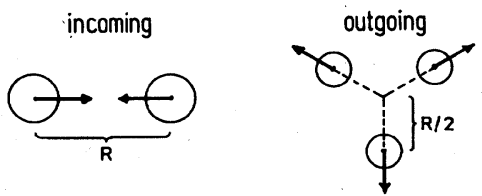

FIG. 6. The geometry of a nuclear reaction leading to fully symmetric three-body breakup.

look briefly at the consequences of an immediate ternary breakup of the compound nucleus. Reactions of this kind have been observed ${ }^{20}$ in lighter collision systems, e.g., $\mathrm{Kr}+\mathrm{Er}$ and $\mathrm{Xe}+\mathrm{Sn}$ at $12.5 \mathrm{MeV} /$ nucleon. In the following, however, we again consider the system $U+U$ as an example.

We restrict our discussion to a particularly simple case which is depicted in Fig. 6: The incoming path of the two nuclei is a Rutherford trajectory of zero angular momentum up to the distance of closest approach. After a time interval $T$ three fragments of equal mass $\left(\frac{1}{3} A_{\text {tot }}\right)$ separate from each other in a fully symmetric breakup (relative angles $\vartheta=120^{\circ}$ in the center of mass system). Due to the chosen special geometry this motion is equivalent to the separation of two nuclei with modified kinematical conditions if one restricts attention to the monopole part of the Coulomb potential seen by the electrons. For reasons of energy conservation the starting point for the outgoing trajectory, $2 \widetilde{a}$, has to be larger than $2 a$. If energy loss is

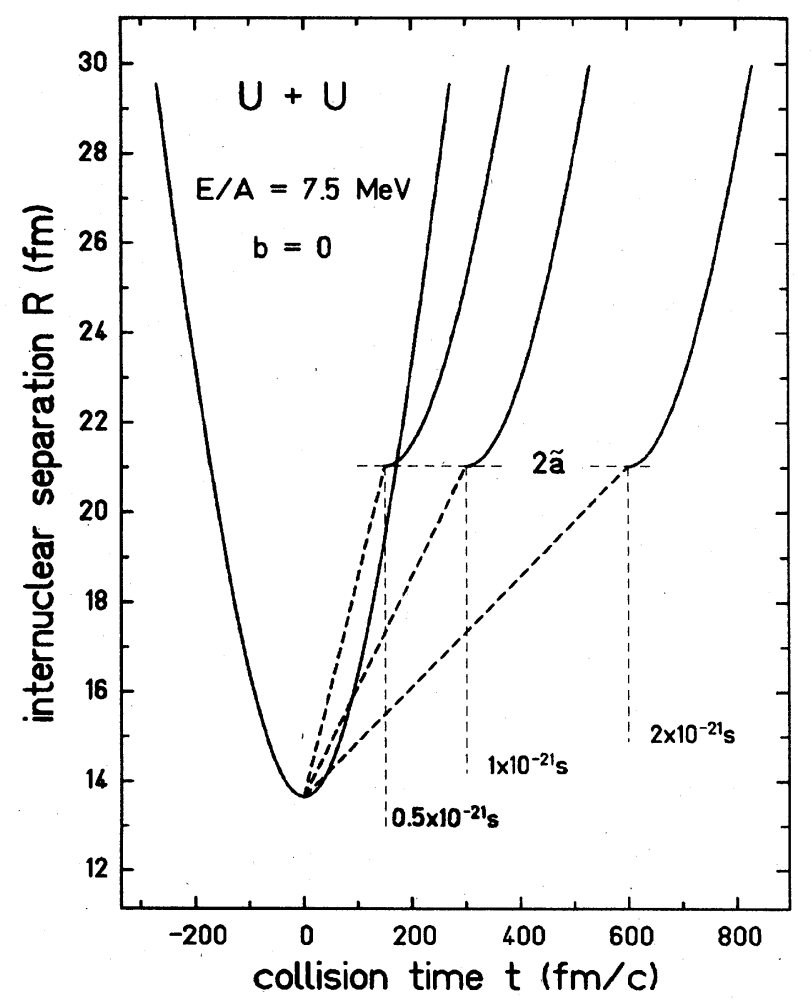

FIG. 7. Schematic model for the nuclear trajectory $R(t)$ in a central $U+U$ collision assuming three-body breakup according to Fig. 6. Three values for the reaction time $T$ have been assumed.
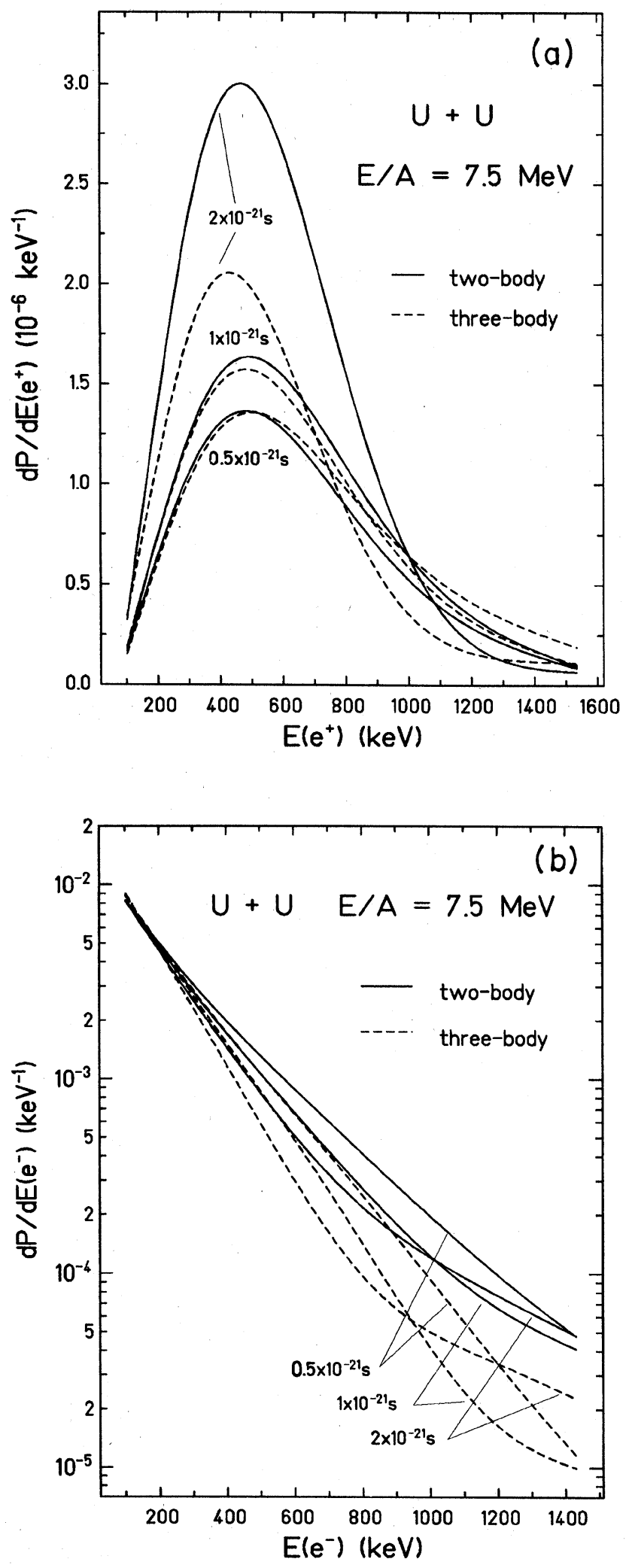

FIG. 8. The calculated spectra for positron (a) and $\delta$-electron (b) emission in central $7.5 \mathrm{MeV} /$ nucleon reactions. Results for two-body (full lines) and symmetric three-body (dashed lines) decay of the compound system are compared, using the schematic trajectories shown in Fig. 7. 
neglected the outgoing motion is described by a modified Rutherford trajectory $R(t)$ with the replacements

$$
a \rightarrow \widetilde{a}=\frac{8}{9} \sqrt{3} a, \quad Z \rightarrow \widetilde{Z}=\frac{2}{3}(2 \sqrt{3})^{1 / 2} Z .
$$

The nuclear reaction time $T$ is used as a free parameter to match both parts of the trajectory (cf. Fig. 7) assuming, quite arbitrarily, linear motion during the reaction.

In Fig. 8(a) we compare positron spectra from the twobody breakup $(2 B)$ (full lines) and from the ternary breakup $(3 B)$ (dashed lines) for three different reaction times $T=0.5,1$, and $2 \times 10^{-21} \mathrm{~s}$. As expected, $2 B$ yields higher positron production probabilities for larger reaction times due to the constant larger spontaneous decay width. For smaller times $T$, however, $3 B$ exceeds $2 B$ in the highenergy region, due to the high radial velocity during the breakup $[\dot{R}=(2 \widetilde{a}-2 a) / T]$ leading to an increased induced emission of positrons.

Figure 8(b) shows $\delta$-electron spectra for the same kinematical conditions. Obviously for the reaction times shown, spectra from the $2 B$ case always exceed those from the ternary breakup. If the model were applied for even smaller values of $T$ we expect more induced excitations in the $3 B$ case due to the higher radial velocity $\dot{R}$ during the reaction.

Although the steep falloff of the $3 B$ electron spectra looks quite remarkable, definite theoretical predictions require a more refined dynamical model. If the result of Fig. 8 is confirmed, the measurement of $\delta$-electron spectra may complement the nuclear physics methods to investigate the ternary breakup process, especially when applied to somewhat lighter collision systems.

\section{CONCLUSIONS}

We have studied the mechanisms of $\delta$-electron emission and pair production in deep-inelastic collisions of very heavy ions within the framework of a semiclassical theory of excitation based on the quasimolecular picture. The theory properly takes into account the resonance character of the "dived" $1 s$ state. ${ }^{3}$ The electron-electron interaction is included within an adiabatic approximation to the time-dependent Hartree-Fock-Slater formalism. ${ }^{4}$

In the semiclassical model the atomic excitation probabilities are affected by the time development of the internuclear distance $R(t)$ and velocity $d R / d t$ during the reaction. We have studied the $\delta$-electron and positron spectra in $\mathbf{U}+\mathbf{U}$ collisions for nuclear trajectories resulting from two friction models, ${ }^{8,12}$ in comparison with elastic Rutherford scattering. Theoretical results were compared with experimental data of Backe et al. ${ }^{14}$ for $\mathrm{U}+\mathrm{U}$ at bombarding energies close to and well above the Coulomb barrier $(5.9,7.5$, and $8.4 \mathrm{MeV} /$ nucleon), where the measurement of $\delta$-electron and positron spectra seems to be an appropriate tool for drawing conclusions on the nuclear motion during the inelastic heavy-ion reaction.
Indeed, the experimental results show clear deviations from the predictions obtained under the assumption of pure Coulomb scattering. For the $\delta$-electron spectra, model $\mathrm{I},{ }^{8}$ based on the nuclear proximity prescription, fits the experimental data for $7.5 \mathrm{MeV} /$ nucleon bombarding energy quite nicely on the absolute scale. This, however, relies on the knowledge of a normalization factor. Furthermore, the agreement is less satisfactory at the higher bombarding energy of $8.4 \mathrm{MeV} /$ nucleon. Model II, ${ }^{12}$ which accounts for neck formation and predicts larger reaction times, is more appropriate to agree with the slope of the measured spectra. The need for different renormalization of the theoretical results, however, is not yet understood.

In the case of positron emission the experimental errors are much larger due to poor statistics and background subtraction. Keeping this in mind, the experimental positron data seem to contradict both nuclear reaction models. This would call for a more severe modification of the nuclear trajectories than provided by the two reaction models under consideration. Larger delay times of about $2 \times 10^{-21} \mathrm{~s}$ may be deduced from a comparison between experiment and theoretical positron yields.

However, it is obvious from a comparison between results obtained within the schematic trajectory model, ${ }^{5-7}$ where $R(t)$ was kept fixed for a time interval $T$, and the results discussed in this paper that atomic excitation processes are influenced not only by the nuclear time delay of the reaction but also by the shape of the nuclear trajectory. Severe interferences between different excitation processes take place and an interpretation in terms of a single parameter is insufficient. A further complication is added by the fact that the experiment measures only an average over many different trajectories. These problems clearly call for an experimental determination of the final state of the nuclear reaction in detail, in particular by fixing the $Q$ value $^{19,21}$ or by selecting different types of reaction events (sequential fission of one or of both nuclei to favor different regions of impact parameters ${ }^{22}$ ). Then experiments measuring the emission of $\delta$ electrons and positrons (which is feasible in very heavy systems with $Z>160$ ), possibly taken together with inner-shell vacancy formation, ${ }^{5,19}$ may play the role of an arbiter distinguishing between various nuclear reaction models.

\section{ACKNOWLEDGMENTS}

We acknowledge very fruitful discussions with $\mathbf{H}$. Backe, U. Gollerthan, E. Kankeleit, R. Krieg, H. Oeschler, and P. Senger concerning their experiment. This work was supported by the Bundesministerium für Forschung und Technologie (BMFT) and the Deutsche Forschungsgemeinschaft (DFG). One of us (G.S.) acknowledges the support of the DFG-Heisenberg Programm. 
${ }^{1}$ G. Soff, W. Greiner, W. Betz, and B. Müller, Phys. Rev. A 20, 169 (1979).

${ }^{2}$ G. Soff, J. Reinhardt, B. Müller, and W. Greiner, Z. Phys. A 294, 137 (1980).

3J. Reinhardt, B. Müller, and W. Greiner, Phys. Rev. A 24, 103 (1981).

${ }^{4}$ T. H. J. de Reus, J. 'Reinhardt, B. Müller, W. Greiner, G. Soff, and U. Müller, J. Phys. B 17615 (1984).

${ }^{5}$ U. Müller, J. Reinhardt, G. Soff, B. Müller, and W. Greiner, Z. Phys. A 297, 357 (1980).

6J. Reinhardt, U. Müller, B. Müller, and W. Greiner, Z. Phys. A 303, 173 (1981); U. Müller, G. Soff, T. de Reus, J. Reinhardt, B. Müller, and W. Greiner, ibid. 313, 263 (1983).

${ }^{7}$ G. Soff, J. Reinhardt, B. Müller, and W. Greiner, Phys. Rev. Lett. 43, 1981 (1979).

8J. R. Birkelund, L. E. Tubbs, J. R. Huizenga, J. N. De, and D. Sperber, Phys. Rep. 56, 107 (1979).

9J. BYocki, J. Randrup, W. J. Swiatecki, and C. F. Tsang, Ann. Phys. (N.Y.) 105, 427 (1977).

10J. Randrup, Ann. Phys. (N.Y.) 112, 356 (1978).

11J. P. Bondorf, M. I. Sobel, and D. Sperber, Phys. Rep. 15C, 83 (1974).

12R. Schmidt, V. D. Toneev, and G. Wolschin, Nucl. Phys. A311, 247 (1978).

${ }^{13}$ U. Müller, Ph.D. thesis, University of Frankfurt, 1984.
${ }^{14}$ H. Backe, P. Senger, W. Bonin, E. Kankeleit, M. Krämer, R. Krieg, V. Metag, N. Trautmann, and J. B. Wilhelmy, Phys. Rev. Lett. 50, 1838 (1983).

${ }^{15}$ H. Freiesleben, K. D. Hildenbrand, F. Pühlhofer, W. F. W. Schneider, R. Bock, D. v. Harrach, and H. J. Specht, Z. Phys. A 292, 171 (1979).

${ }^{16} \mathrm{U}$. Gollerthan, private communication.

${ }^{17}$ P. Glässel, D. v. Harrach, Y. Civelekoglu, R. Männer, H. J. Specht, J. B. Wilhelmy, H. Freiesleben, and K. D. Hildenbrand, Phys. Rev. Lett. 43, 1483 (1979).

18P. Senger, Ph.D. thesis, Technische Hochschule Darmstadt, 1983.

${ }^{19}$ Ch. Stoller, M. Nessi, W. Wölfli, W. E. Meyerhof, J. D. Molitoris, E. Morenzoni, E. Grosse, and Ch. Michel, Gesellschaft für Schwerionenforschung Report GSI 83-1, 1983, p. 19; XIII ICPEAC, Abstracts, 1983, p. 438; and submitted to Phys. Rev. Lett.

${ }^{20}$ P. Glässel, D. v. Harrach, H. J. Specht, and L. Grodzins, Z. Phys. A 310, 189 (1983).

${ }^{21}$ F. Güttner, W. Koenig, N. Lutz, B. Martin, H. Skapa, J. Soltani, H. Banda, A. V. Ramayya, F. Bosch, and Ch. Kozhuharov, XIII ICPEAC, Abstracts, 1983, p. 439.

${ }^{22}$ U. Gollerthan, E. Kankeleit, R. Krieg, H. Oeschler, and P. Senger, private communication. 